\title{
Tratamiento con linezolid intraventricular más intravenoso en un paciente pediátrico con ventriculitis por Enterococcus faecium resistente a vancomicina
}

\author{
Treatment with intraventricular linezolid plus intravenous in a pediatric \\ patient with vancomycin-resistant Enterococcus faecium ventriculitis
} \author{
Jorge Mario Chávez Estrada, ${ }^{\S}$ Napoleón González Saldaña*

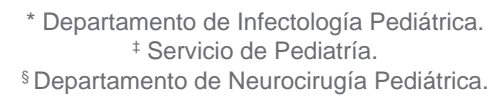 \\ Instituto Nacional de Pediatría, Ciudad de México.
}

Nancy Evelyn Aguilar Gómez,* Sairy Natalia Pérez Murillo,* Tania María Vargas Vázquez,

RESUMEN

\begin{abstract}
La ventriculitis es una complicación grave observada posterior a procedimientos neuroquirúrgicos y se asocia, en la mayoría de los casos, a la presencia de dispositivos de drenaje ventricular externo. Los principales agentes etiológicos son microorganismos grampositivos como Staphylococcus epidermidis y Staphylococcus aureus; sin embargo, en la actualidad se ha observado un aumento en las infecciones por Enterococcus spp. en el ámbito hospitalario, asociado a diferentes patrones de resistencia antimicrobiana. La emergencia de infección por enterococo resistente a vancomicina (ERV) en pediatría se ha convertido en un reto terapéutico y se asocia a una elevada mortalidad.
\end{abstract}

Palabras clave: Linezolid, vancomicina, ventriculitis, Enterococcus faecium, resistencia antimicrobiana.

\section{INTRODUCCIÓN}

La ventriculitis es una complicación grave observada posterior a procedimientos neuroquirúrgicos y se asocia, en la mayoría de los casos, a la presencia de dispositivos de drenaje ventricular externo. La incidencia mundial varía de 4 a 17\%., ${ }^{1,2}$ Los principales agentes etiológicos son microorganismos

Financiamiento: Ninguno.

Conflicto de intereses: Ninguno.

https://dx.doi.org/10.35366/94420

Rev Latin Infect Pediatr 2020; 33 (2): 92-96
ABSTRACT

Ventriculitis is a serious complication observed after neurosurgical procedures and is associated in most cases with the presence of external ventricular drainage devices. The main etiologic agents are Gram positive microorganisms such as Staphylococcus epidermidis and Staphylococcus aureus, however, an increase in Enterococcus spp. Infections has now been observed. in the hospital setting, associated with different patterns of antimicrobial resistance. The emergence of vancomycin-resistant Enterococcal $(E R V)$ infections in pediatrics has become a therapeutic challenge and is associated with high mortality.

Keywords: Linezolid, vancomycin, ventriculitis, Enterococcus faecium, drug resistance.

grampositivos como Staphylococcus epidermidis y Staphylococcus aureus en $80 \%$ de los casos. ${ }^{2}$ Otros microorganismos menos frecuentes son: bacilos gramnegativos aerobios y ocasionalmente hongos. $^{2}$

En la actualidad, se ha observado un aumento en las infecciones por Enterococcus spp. en el ámbito hospitalario (con diferentes patrones de resistencia), lo cual se convierte en un reto terapeútico, ya que se reporta una mortalidad de $21-25 \% .^{1,3}$ Linezolid es una de las opciones terapéuticas disponibles; actualmente se han documentado algunos reportes de casos de enterococo resistente a linezolid. ${ }^{2}$ 
Rev Latin Infect Pediatr 2020; 33 (2): 92-96

El uso de antibióticos intratecales ha incrementado en el tratamiento de pacientes con ventriculitis en los últimos años. La indicación es por la inhabilidad de esterilizar el líquido cefalorraquídeo a pesar del tratamiento antimicrobiano específico por vía intravenoso. La eficacia y seguridad de la administración de estos medicamentos se ha descrito en el transcurso de los años. Sin embargo, la utilización y el perfil de seguridad de las nuevas generaciones de antibióticos vía intraventricular permanece desconocida. ${ }^{4}$

A continuación se describe el caso de un paciente pediátrico con ventriculitis asociada a sistema de derivación ventriculoperitoneal por Enterococcus faecium intermedio a linezolid tratado exitosamente con linezolid intravenoso e intraventricular.

\section{CASO CLÍNICO}

Lactante femenino de nueve meses de edad con antecedente de prematurez de 24 semanas de gestación, apneas mixtas, retinopatía del prematuro, displasia broncopulmonar, atelectasias persistentes y hemorragia intraventricular grado IV que ameritó colocación de sistema de derivación ventriculoperitoneal (SDVP). Ingresó el 12 de junio de 2019 por presentar hipoactividad, somnolencia y crisis convulsivas tonicoclónicas generalizadas acompañadas con fiebre de $39{ }^{\circ} \mathrm{C}$. Se realizó tomografía axial computarizada (TAC) simple de cráneo y como hallazgo se encontró imagen hipodensa localizada en asta frontal y temporal del ventrículo lateral izquierdo, correspondiente a neumoencéfalo.

La paciente tiene antecedente de múltiples intervenciones de recambio de sistemas y ventriculostomía, la última se realizó el 08 de mayo de 2019. Ante sospecha de neuroinfección se puncionó reservorio del sistema ventriculoperitoneal, en el cual se obtuvo líquido cefalorraquídeo (LCR) de aspecto turbio con reporte citoquímico y citológico con proteínas de $243 \mathrm{mg} / \mathrm{dL}$, glucosa de $38 \mathrm{mg} / \mathrm{dL}$, células 400 $\mathrm{mm}^{3} / \mathrm{mL}$, polimorfonucleares $20 \%$, mononucleares $80 \%$. Con diagnóstico de ventriculitis bacteriana por presentación clínica y reporte de citoquímico se inició cobertura antibiótica con ceftriaxona y vancomicina en espera del resultado del cultivo. El día 13 de junio de 2019 se retiró el SDVP y se dejó con drenaje ventricular externo. Poco después se inició terapia con meropenem intravenoso a altas dosis por cultivo de LCR en que se aisló Klebsiella pneumoniae productor de betalactamasa de espectro extendido, y fluconazol intravenoso por aislamiento de Candida albicans.

Durante los siguientes 10 días la paciente continuó con citoquímico alterado y fiebre persistente, se tomaron nuevos cultivos en los que se reportó aislamiento de Enterococcus faecium con gen vanA positivo en LCR. Se agregó linezolid intravenoso, se continuó por 30 días sin mejoría clínica, ya que los cultivos de LCR extraídos cada 48 horas no lograron mostrar la eliminación de Enterococcus faecium, a pesar de la cobertura dirigida. Considerando la persistencia de la infección, el deterioro neurológico de la paciente y la imposibilidad para realizar recambio de la derivación externa, se tomó la decisión de agregar linezolid intraventricular, rescate a dosis de $10 \mathrm{mg} / \mathrm{kg} /$ día cada 24 horas, ajustado a capacidad ventricular máxima como medida de rescate ante la mala evolución de la paciente.

A los cinco días de iniciada la terapia intraventricular se inició a tener mejoría de citológico y citoquímico. Al concluir el tratamiento (15 días de intraventricular) la paciente se encontró clínicamente afebril, sin deterioro neurológico, con citoquímico, citológico normales, y se tomaron nuevos cultivos, los cuales nuevamente se reportaron negativos. Se retiró ventriculostomía y se colocó nuevo SDVP. La paciente permaneció estable, por lo que se egresó sin datos clínicos o microbiológicos de infección.

En la Tabla 1 podemos observar la evolución microbiológica y el tratamiento realizado en esta paciente.

\section{DISCUSIÓN}

El enterococo es un microorganismo oportunista y actualmente es uno de los principales patógenos de adquisición hospitalaria a nivel mundial. ${ }^{5} \mathrm{La}$ transmisión ocurre por contacto directo y puede sobrevivir en las manos del personal médico hasta por 30 minutos. ${ }^{6}$ En las últimas dos décadas la emergencia de cepas de enterococo resistente a la vancomicina ha sido alarmante, ocurriendo a nivel mundial de $12-35 \%$ y se ha asociado a una elevada mortalidad. $5,7,8$

Las opciones de tratamiento para E. faecium resistente a vancomicina en niños es limitada, lo cual se convierte en un reto terapéutico, ya que la mayoría de los antibióticos disponibles no alcanzan concentraciones terapéuticas en LCR. 
Tabla 1: Evolución de líquido cefalorraquídeo, cultivos y tratamiento indicado en esta paciente.

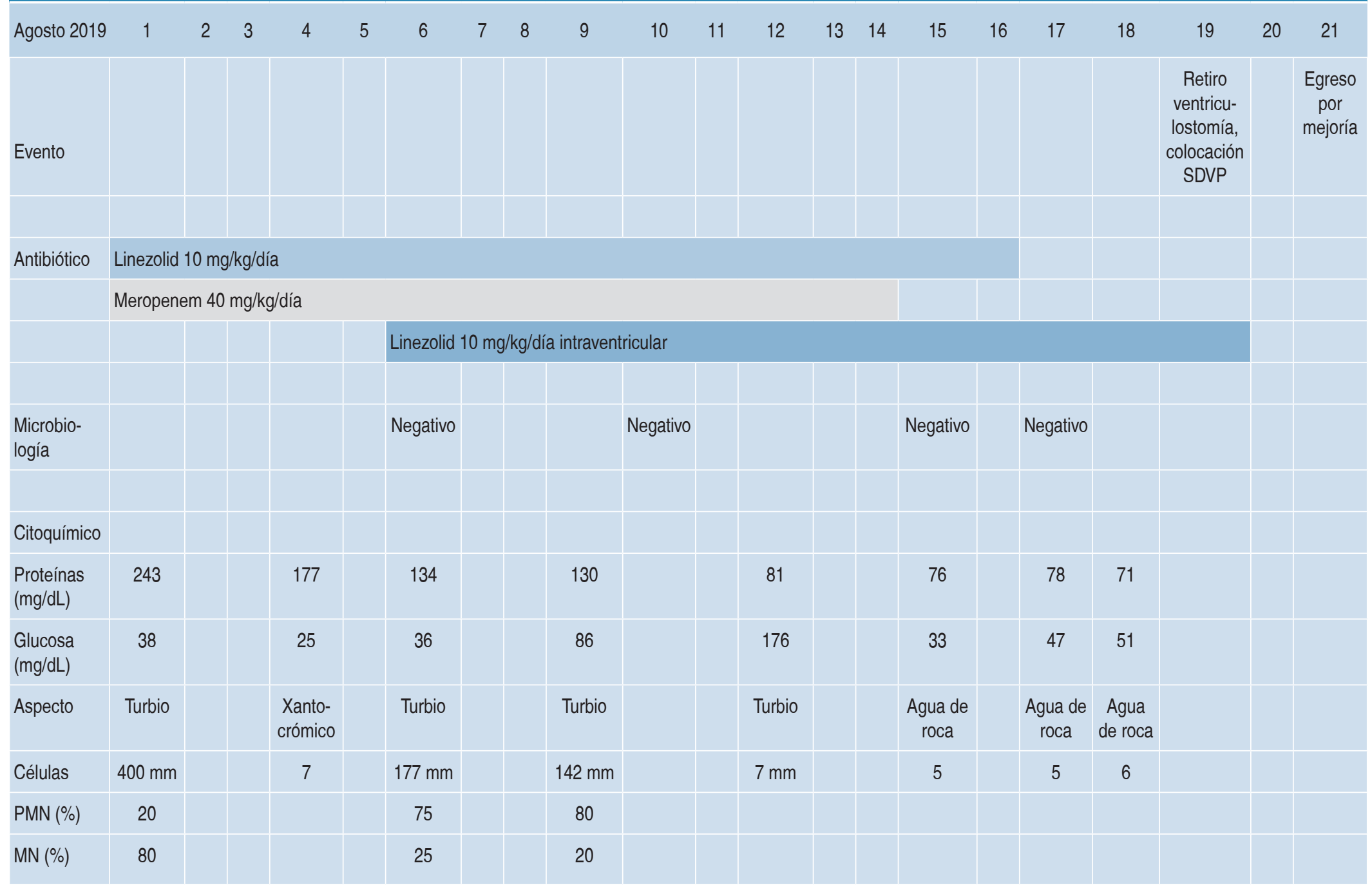


Rev Latin Infect Pediatr 2020; 33 (2): 92-96

No existen ensayos clínicos aleatorizados que incluyan el tratamiento óptimo de las infecciones asociadas a sistemas de derivación ventriculoperitoneal. El tratamiento incluye retiro del dispositivo, drenaje externo, antibióticos parenterales y reemplazo del sistema de derivación una vez que se obtenga esterilización del LCR. Si no se puede realizar el retiro del dispositivo y no se consigue mejoría clínica, a pesar del tratamiento intravenoso, los antibióticos intraventriculares pueden ser de utilidad. ${ }^{3,9}$

En este caso, siguiendo las recomendaciones de la Infectious Diseases Society of America (IDSA), se dio tratamiento antimicrobiano intraventricular en combinación con el intravenoso, ya que la paciente no había respondido de forma adecuada a la terapéutica antimicrobiana intravenosa, persistía con deterioro neurológico, debido a las comorbilidades no era candidata a nuevo tratamiento quirúrgico para recambio del sistema de derivación ventricular, y persistía con alteraciones en las características citoquímicas y citológicas del LCR.

Ningún antibiótico ha sido aprobado por la Food and Drug Administration (FDA) para uso ventricular y su uso está basado en los reportes de caso a nivel mundial. La mayor parte de la experiencia clínica se ha observado con vancomicina y gentamicina; el uso de otros agentes sólo se ha limitado a reportes de caso. Hoy en día, se han utilizado en la práctica clínica amikacina, colistina, daptomicina, polimixina $B$, quinupristina/dalfopristina, tobramicina y vancomicina. ${ }^{10,11} \mathrm{~A}$ pesar de la efectividad de linezolid se encuentran pocos estudios sobre su uso intraventricular. ${ }^{4}$ Se ha utilizado con éxito para infecciones por $E$. faecium en niños de forma intravenosa. ${ }^{8}$

Linezolid es una oxazolidinona que actúa inhibiendo la síntesis proteica evitando la formación del complejo de iniciación. ${ }^{12}$ Es utilizada para el tratamiento de infecciones graves por microorganismos grampositivos resistentes a la vancomicina. ${ }^{12}$ Fue aprobada por la FDA para uso en niños en $2002 .{ }^{8}$ Linezolid se ha utilizado con éxito de forma intravenosa en varios pacientes con ventriculitis asociada a SDVP; sin embargo, no es considerada la primera línea de tratamiento y se reserva para pacientes con infección multidrogorresistente. ${ }^{1}$ Linezolid se une poco a las proteínas plasmáticas $(30 \%)$ y alcanza excelente penetración en LCR (aproximadamente 66\%) con una relación de LCR/ suero de 0.92. ${ }^{13}$
Se han publicado reportes de caso de tratamiento exitoso con linezolid para Staphylococcus y Enterococcus spp. resistente a vancomicina en infecciones de sistema nervioso central, a pesar de que el linezolid no es bactericida. ${ }^{13}$ Graham y colaboradores fueron los primeros en reportar el uso de linezolid en un paciente pretérmino con un SDVP y ventriculitis causada por $E$. faecium resistente a ampicilina y vancomicina. Langgartner y colegas $^{12}$ describieron cinco casos de infección relacionada a ventriculostomía en niños pretérmino tratados con éxito con linezolid a 10-15 mg/kg IV cada ocho horas por 14-16 días. Todos los estudios reportados tuvieron una duración de tratamiento de 15-21 días, obtuvieron mejoría clínica y esterilización de cultivos con el tratamiento intravenoso y no reportaron efectos adversos. $^{12}$

En el caso de nuestra paciente se utilizó linezolid intraventricular, el cual se ha descrito en pocos reportes de casos en la literatura previamente. ${ }^{4}$ Uno de ellos reportado por Lich y colaboradores, en 2016, en una paciente de 32 años con alergia severa a la vancomicina, posterior a seis días con terapia intraventricular con linezolid $10 \mathrm{mg}$ cada 24 horas se obtuvo esterilización del LCR y no presentó eventos adversos. ${ }^{4}$ En el caso de nuestra paciente a los cinco días de iniciada la terapia intraventricular se inició a tener mejoría de citológico y citoquímico, los cuales fueron de características normales para el día siete de tratamiento.

La resistencia de linezolid en enterococo resistente a vancomicina se ha reportado previamente. La disminución en la susceptibilidad a linezolid posterior a la exposición de este medicamento se ha reportado previamente como resultado de una mutación genética en el gen 23SrRNA. ${ }^{12}$ En 2001 González y colegas reportaron casos de enterococo resistente a vancomicina y linezolid aislados de diferentes sitios en cinco pacientes de tres hospitales en tres meses. En 2002 se reportó el primer caso de enterococo resistente a linezolid y vancomicina en UK. Y similares aislamientos se han reportado en todo el mundo, por ejemplo en Alemania, EUA y Brasil.

En nuestra paciente se documentó resistencia intermedia a linezolid in vitro, lo cual consideramos fue resultado de la exposición previa a linezolid intravenoso.

No existe un algoritmo ideal para el tratamiento por las ventriculitis por enterococo. El tratamiento en estos casos es un reto. La duración del tratamiento 
antimicrobiano no está completamente definido y es dependiente del microorganismo aislado, la negativización de cultivo y ocasionalmente de los hallazgos de LCR. ${ }^{9}$ En este caso, la duración del tratamiento se llevó a cabo tomando en cuenta el criterio de 10-14 días posteriores al último cultivo positivo que se documentó en el paciente.

En nuestro conocimiento éste es el primer reporte en México de aislamiento de Enterococcus faecium intermedio a linezolid y resistente a vancomicina en líquido cefalorraquídeo de un sistema de derivación ventriculoperitoneal infectado en pediatría.

\section{CONCLUSIÓN}

La resistencia a linezolid de Enterococcus faecium resistente a vancomicina es rara y es un reto el tratamiento. Este caso enfatiza la urgente necesidad de ensayos clínicos en pediatría para ayudar a establecer el beneficio de linezolid en el tratamiento de ventriculitis, así como el tratamiento antibiótico de microorganismos resistentes emergentes. También ofrece una guía clínica del uso de linezolid IV o intraventricular en niños si no se tienen mejores alternativas.

\section{REFERENCIAS}

1. Irfan S, Qamar S. A case report on isolation of linezolid and vancomycin-resistant Enterococcus faecium species from cerebrospinal fluid of a patient suffering from ventriculoperitoneal shunt-associated meningitis. Cureus. 2019; 11 (9): 1-4.

2. Humphreys $\mathrm{H}$, Jenks J. Surveillance and management of ventriculitis following neurosurgery. J Hosp Infect. 2015; 89 (4): 281-286.

3. Sahin A, Dalgic N. Intraventricular plus intravenous tigecycline for the treatment of daptomycin nonsusceptible vancomycin-resistant enterococci in an infant with ventriculoperitoneal shunt infection. World Neurosurg. 2019; 130: 470-473.

4. Lich B, Conner A, Burks J, Glenn C, Sughrue M. Intrathecal/ intraventricular linezolid in multidrug-resistant Enterococcus faecalis ventriculitis. J Neurol Surg Rep. 2016; 77: 160-161.

5. Raza T, Ullah SR, Mehmood K, Andleeb S. Vancomycin resistant enterococci: a brief review. J Pak Med Assoc. 2018; 68 (5): 767-772.

6. Sood S, Malhotra M, Das BK, Kapil A. Enterococcal infections \& antimicrobial resistance. Indian J Med Res. 2008; 128: 111-121.

7. Wang G, Yu F, Lin H, Murugesan K, Huang W, Hoss AG et al. Evolution and mutations predisposing to daptomycin resistance in vancomycin-resistant Enterococcus faecium ST736 strains. PLoS One. 2018; 13: e0209785.

8. Jaspan $\mathrm{H}$, Brothers A, Campbell A, McGuire J, Browd S, Manley $\mathrm{T}$ et al. Multidrug-resistant Enterococcus faecium meningitis in a toddler. Pediatr Infect Dis J. 2010; 29 (4): 379-381.

9. Tunkel A, Hasbun R, Bhimraj A, Byers K, Kaplan S, Scheld W, Van de Beek D et al. Infectious diseases Society of America's Clinical practice guidelines for healthcareassociated ventriculitis and meningitis. Clin Infect Dis. 2017; 64: 701-706.

10. Patel T, Lewis M, Nielsley M, Chowdhury M. Postneurosurgical central nervous system infection due to Enterococcus faecalis successfully treated witj intraventricular vancomycin. Infect Dis Clin Pract. 2016; 24: 174-176.

11. Quinn A, Parada J, Belmares J, O'Keefe J. Intrathecal colistin and sterilization of resistant Pseudomonas aeruginosa shunt infection. Ann Pharmacother. 2005; 39 (5): 949.

12. Chiappini E, Conti C, Galli L, Martino M. Clinical efficacy and tolerability of linezolid in pediatric patients: a systematic review. Clin Ther. 2010; 32 (1): 1-23.

13. Myrianthefs P, Markantonis S, Vlachos K, Anagnostaki M, Boutzouka E, Panidis D et al. Serum and cerebrospinal fluid concentrations of linezolid in neurosurgical patients. Antimicrob Agents Chemother. 2006; 50 (12): 3971 . 3976.

Correspondencia:

Nancy Evelyn Aguilar Gómez

E-mail: nancyevelyn_061004@hotmail.com 